\author{
Military Technical College \\ Kobry El-Kobba \\ Cairo, Egypt
}

12-th International Conference

on

Aerospace Sciences \&

Aviation Technology

\title{
IMPROVING LEO SATELLITE COMMUNICATION COVERAGE AREA BY USING LEO-GEO CROSS-LINK
}

\author{
E.A Soleit *, M. A. Soliman ** and Karim A. Fouad *
}

\begin{abstract}
This paper presents a proposal for a data relay technique, which utilizes an existing Geo-stationary Orbit (GEO) communication satellite as a data relay satellite, in cooperation with Low Earth Orbit (LEO) satellite. The proposed scheme can increase the communication availability with the remote sensing LEO satellite. Moreover, the proposed system improves both the communication coverage area of the LEO satellite and its scanning availability in the real time. This improvement is a real need in the remote sensing applications.
\end{abstract}

\section{KEY WORDS}

Geo-Stationary Orbit (GEO), Low Earth Orbit (LEO), data relay satellite, remote sensing, communication coverage area.

\footnotetext{
* Egyptian Armed Forces
}

** Zaqziq University 


\section{INTRODUCTION}

A remote sensing satellite possesses a payload as a multi-spectral imager. The extracted images are scanned; processed and stored on a mass storage media. The processed data and its related information are transmitted to the ground station through downlink, when the LEO satellite ground track lies inside the ground station coverage area. The duration of the communication availability between the LEO satellite and ground station can be deduced according to the LEO satellite height and its orbit parameters. This communication period constitutes a critical design parameter for the choice of communication architecture, mission analysis and design of remote sensing satellites [1, 2, 3].

American and European trends for using Tracking and Data Relay Satellite Systems (TDRSS) are very expensive to be accessed by other countries like Egypt. It may not be released to share these systems with other parties [2].

The availability of GEO communication satellite as the Egyptian satellite [NILE SAT] grows up the idea of utilizing its capability as a relay communication satellite as shown in Figure 1.

So instead of down linking the data to the ground station during the short period of communication availability between the LEO satellite and the ground station, the LEO remote sensing satellite will uplink this data to the GEO satellite for a longer period of communication between GEO and LEO satellites. The ratio between the communication periods in both cases is equal to the ratio between the coverage areas of the ground station to that of the GEO satellite [4].

This paper includes four sections. Section two presents calculation of the parameters required to establish a cross-link power budget between GEO and LEO satellites. Due to the fact that the distance between the LEO satellite and the ground station is much less than the distance between LEO satellite and GEO satellite, the power required for establishing communication link between the LEO and the GEO satellites is greater than the one required for LEO-ground station communication. The tracking angles of LEO satellite are also calculated where a LEO satellite antenna designed to track the GEO satellite [11]. This antenna should move according to specific maneuver that is controlled by an algorithm developed to calculate these angles, which the antenna should follow in both azimuth and elevation planes.

Section three deals with the developed algorithms analysis and simulation results. In section four, a conclusion of the results and analysis is presented besides presenting practical application for the proposed system.

\section{CROSS-LINK POWER BUDGET}

A narrow beam antenna is used for LEO - GEO communication link in order to increase the gain and to minimize the power needed for communication between both satellites. The range between the GEO and the LEO satellites is calculated on the boundaries of the required coverage area. In order to design the required link, the GEO satellite coverage area has to be determined and plotted. The NILESAT is taken as an example for the GEO satellite. Its coverage area is depicted in Figure 2. When the LEO satellite crosses the GEO coverage area, the tracking for the GEO satellite takes place and the LEO antenna starts to align with the path of the GEO 
satellite. The maximum and minimum distance between the two satellites on each track should be calculated and an optimization is needed in order to get a mean value. Taking the area of interest (ground track sample), it might be looked in a simple way as In Fig. 3. The ground track sample was one of the ground tracks that pass through the coverage area of the NILESAT satellite.

Figure 4 represents the GEO -LEO locations w .r .t Earth. It is illustrated in order to deduce the relative motion relationship between the two satellites.

From the geometry in fig. 4 ;

$$
S=\sqrt{r^{2}-2 r R \cos \beta_{0}+R^{2}}
$$

But in case of LEO -GEO cross-link (shown in the dashed lines)

$S=\sqrt{r^{2}-2 r\left(R+H_{l e o}\right) \cos \beta_{0}+\left(R+H_{l e o}\right)^{2}}$

Propagation Path Length

From spherical triangle properties [5],

$\cos \beta_{0}=\cos \phi \cos \Delta \lambda$

Where

$\beta_{0} \ldots \ldots$. The angle between the GEO satellite nadir vector and the LEO satellite nadir vector

$\boldsymbol{\varphi} . . . . .$. Latitude of LEO satellite in radians

$\Delta \lambda \ldots . .$. difference between GEO and LEO longitude in radians

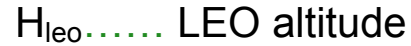

$\mathrm{H}_{\text {geo }} \ldots . .$. GEO altitude

R......... Earth radius

The values of the propagation path length are considered effective in calculating the space loss due to the propagation path as stated in equation (3) [6].

$L=20 \log S+20 \log f+92.45$

By substitution for the calculated values for $S_{\max }, S_{\min }$, and at frequency $(f)=17 \mathbf{G H Z}$,

$L_{\max }=388.85 \mathrm{dbw}$

$L_{\text {min }}=387.85 \mathrm{dbw}$

From the above results, there is no need to get $S_{\text {mean }}$ since the losses in both cases (maximum, minimum) are approximately the same.

Matlab program is developed that uses different approximate loops for the transmit power, antenna diameter, and beam width $P, D, \theta$ respectively. Their optimum values can be deduced based on the resulting figures.

So;

$$
W_{f}=E I R P-20 \log S-71.0
$$

\section{Where}

$W_{f} \ldots . .$. Power flux density $=-96 \mathrm{dbw}$

EIRP........Effective Isotropic Radiated Power $=55.038 \mathrm{dbw}$ [NILESAT]

S. .Propagation path length

$$
G_{t}=-10 \log P+E I R P
$$




\section{Where}

$\mathrm{G}_{\mathrm{t}}$.... transmit antenna gain

$10 \log P=E I R P+20 \log \theta+12\left(\frac{e}{\theta}\right)^{2}-44.3$

$G_{t}=-159.5+20 \log D+20 \log f+10 \log \eta$

$10 \log \left(\frac{P}{D^{2}}\right)=E I R P+159.9-20 \log f-10 \log \eta$

\section{Where}

e............ can be expressed in terms of $\theta$ as $e=\alpha \times \theta$ and $0<\alpha<1$

$\eta$.............antenna efficiency (usually in order of 0.5 )

The energy per bit to noise ratio, which is considered as one of the figures of merit for the link power budget $[5,6]$, can be calculated from the following equation:

$$
E_{b} / N_{0}=E I R P+L_{a}+L_{s}+228.6-10 \log T_{s}-10 \log R
$$

\section{Where}

$\mathrm{R} . \ldots \ldots \ldots \ldots$........ the data rate in bps.

$\mathrm{T}_{\mathrm{s}} \ldots \ldots \ldots \ldots \ldots$ the system noise temperature in Kelvin degrees.

$L_{s} \ldots \ldots \ldots \ldots . . . . . . s p a c e$ loss in $\mathrm{db}$.

$\mathrm{L}_{\mathrm{a}} \ldots \ldots \ldots \ldots . . .$. transmitting antenna loss in $\mathrm{db}$.

In the following figure, the GEO and LEO satellites are located at predetermined heights. Since the GEO satellite position is previously known $\left(7^{0} \mathrm{~W}\right)$ [NILE SAT]. The LEO satellite position is known through on board GPS. From the geometry of the Figure 5, and spherical triangles characteristics, the following equations are derived.

$$
\begin{aligned}
& \cos \beta_{\text {free }}=\cos T_{L} \cos \left(N_{G}-N_{L}\right) \\
& \phi=\tan ^{-1} \frac{G 1 \sin \beta}{G 1 \cos \beta-L 1} \ldots \ldots \ldots . .
\end{aligned}
$$

\section{Where}

$T_{G} \ldots . . . . . . G E O ~ L a t i t u d e$.

$\mathrm{N}_{\mathrm{G}} \ldots \ldots . . . \mathrm{GEO}$ Longitude.

$T_{L} \ldots \ldots \ldots . . . L E O$ Latitude.

$\mathrm{N}_{\mathrm{L}} \ldots \ldots . . . \mathrm{LEO}$ Longitude

L1 .........Distance between earth's center and LEO satellite.

G1 .........Distance between earth's center and GEO satellite.

$B_{\text {free }} . . . . . . .$. the angle between LEO nadir vector and GEO nadir vector (when $T_{G}=0$ )

$\Phi$.........the angle between LEO nadir vector and the line connecting GEO and LEO.

Applying the longitude and latitude ranges (as in fig. 3)in equations (10) and (11) will yield the calculation of angle $(\Phi)$ range over the GEO satellite coverage area. Plotting this range determines the required input for the antenna to achieve such range. 
The angle $(\Phi)$ is the elevation angle of the LEO line of sight to the GEO. the same analysis was done for the other angle $(\Lambda)$, which is the azimuth angle of the LEO line of sight to the GEO, in order to define the required input to the LEO antenna controller.

In the following section, the implementations of the developed algorithms are carried out including illustrations of the resulting responses in each case.

\section{SIMULATION RESULTS}

Simulation programs are developed using Matlab algorithms to obtain the optimal values for the parameters comprising the link power budget. The derived equations are appraised based on the following analysis:

From Figure 3, the following can be deduced:

$\Phi=(60 * \mathrm{pi}) / 180 \ldots \ldots \ldots \ldots \ldots . . .$. latitude in radians.

$\Delta \lambda=\left(30^{*} \mathrm{pi}\right) / 180 \ldots \ldots \ldots \ldots \ldots$ difference in longitude in radians

\section{The following are assumed:}

$\mathrm{H}_{\text {Leo }}=700 \mathrm{~km}$

LEO altitude

$\mathrm{H}_{\text {geo }}=35000 \mathrm{~km}$.

GEO altitude

$\mathrm{R}=6378.14 \mathrm{~km}$

Earth radius

From Figure 4, the following can be deduced:

$r=38000+6378=42000 \mathrm{~km} \ldots$ propagation path length for the GEO satellite.

$\cos \beta_{0}=(\cos \varphi)^{*}(\cos \Delta \lambda) \ldots$... nadir angle measured from sub-satellite point to target.

Substitution by the values of the latitude and longitude of the four corners of the desired coverage area boundaries in equation (2), then substituting the resulting values in equation (1) yields the following:

$$
\begin{gathered}
S_{\max }=39 * 10^{3} \mathrm{~km} \\
S_{\min }=34.7 * 10^{3} \mathrm{~km}
\end{gathered}
$$

\section{The input parameters of the developed program are assigned as follows:}

P... Transmitter power $=5-30$ watts in increments of 5 watts and the calculations will be iterated for each value.

f.......transmitter frequency $=17.3-17.9 \mathrm{GHZ}$ in increments of $0.1 \mathrm{GHZ}$, and as the power parameter, there will be iterative calculations for each value too.

$\theta_{r} \ldots \ldots \ldots \ldots$........

By plotting equations $(6,7,8)$ through iterations for different values of $P$ and $\theta$, different relations between the antenna beam width, antenna diameter, and the transmit

From figures $(6,7,8,9$ and 10$)$ respectively, the optimum values for $P, D, \theta$ can be deduced on condition that they can yield the following results.

- The antenna beam width should be small to achieve high gain.

- If high gain is achieved, this has to be under constraint of the transmitter power parameter, since gain is inversely proportional to the power. That is apparent in Figure 10

- The antenna diameter is also inversely proportional to the antenna beam width as shown in Figure 9. That will be a decisive factor also in choosing the optimal values for the three parameters $[P, \theta, D]$. 
- Since the transmitter is on board of LEO, the TWT should be relatively comparable to the size of the LEO [7]. From similar mission analysis, the appropriate power rating was found to be $\mathbf{2 0}$ watts. That will lead to candidate values of antenna diameter $(D)$, and antenna beam width $(\theta)$ to be $\mathbf{0 . 4 m}$, and $\mathbf{4 . 3 ^ { 0 }}$ respectively. Regarding the losses, it is found that the antenna pointing error is very small and can be negligible as shown from figure (11).

Finally, the Energy per bit $E_{b} / N_{0}$ is calculated using link power budget calculations. It is found to be $11.97 \mathrm{db}$. This value is validated by checking $\left(E_{b} / N_{o}\right)$ reference figure $[4,8]$. This figure is a standard figure used to evaluate the link power budget performance. It is characterized by Bit Error Rate (BER), which is taken as the standard figure of merit for the link power budget. The calculated $E b \mid N_{0}(11.97 \mathrm{db})$ is in range of what its value should be according to the specified BER $\left(10^{-6} \mathrm{bps}\right)[5]$.

For calculating the GEO-LEO tracking angles, equations $(10,11)$ are used to plot the required input to the control system as well as the rates of change of the input, which represents the angular velocity of the tracking antenna. They are represented in fig. 12 and fig. 13. Where the variable angle $(\mathrm{PHI})$ represents the angle $\Phi$ and the variable Rate of PHI represents the rate of change of the angle $\Phi$. The longitude in the figures, represent the sub satellite point of the LEO. From figure 12, it can be concluded that the antenna mounted on the LEO satellite will need a unit step input+ ramp input with the specifications shown in the figure. First it requires a unit step to reach to the initial value in the figure which is $37.5^{\circ}$, and then a negative ramp input is applied so as to reach the final angle $(\Phi)$ value of $33.5^{\circ}$. It should be noted that the communication between the LEO and GEO satellites will be established during the specified range of longitude shown in fig. 12, and 13. The rate of change of angle $(\Phi)$ is constant as shown in the above figure over the range of the specified longitude. It does not exceed $\mathbf{0 . 0 6} \mathbf{~ d e g} / \mathbf{s}$ over the specified longitude range. This rate should be the speed that the antenna will be moving with during that longitude range for angle $(\Phi)$ direction.

In Figure 14, the tracks of the LEO satellite are plotted, where communication is available with the GEO satellite during the bold parts of the plot. The heavy bold area resulted from the boundaries obtained from the NILE SAT coverage area. This area will be compared with another conventional ground station coverage area boundaries located within Egypt. A Matlab Program developed for plotting satellite tracks. This program calculates and plots the tracks of the LEO satellite generally and highlights the areas where the LEO satellite is visible to the GEO satellite. Execution of this program gives the coverage area percentage between the two areas within period of one day time. Based on the latitude and longitude at moment of calculation, the coverage area could vary, but its percentage stays the same [8,9].

The program has calculated the LEO satellite period to be $\mathbf{5 8 8 2}$ seconds i.e. it takes 5882 seconds to complete one rotation around Earth. The calculated available time for communication session with the GEO satellite within the GEO coverage area region is $\mathbf{2 3 8 7}$ seconds.

While the calculated available time for communication in case of typical LEO ground station with the ground station is 185 seconds. So the percentage between 
both cases is $2387 / 185=12.9$. This means that the proposed link has provided an enhancement in communication availability by $\mathbf{1 2 . 9}$ times. Besides, the time that the LEO satellite can send data to the Geo satellite during one period is almost half time of the whole LEO satellite period.

\section{CONCLUSION}

In order to come up with an optimal design for such cross-link that can achieve the best required performance for data relaying between LEO and GEO satellites, A link power budget algorithm is developed to optimize the power budget parameters. Besides, another algorithm is developed for calculating the tracking angles and the LEO-GEO correlation coverage area. A proposed technique using the LEO-GEO relay communication is introduced. The following approaches are recommended as introduced in the following sections:

Establishing such cross-link between LEO-EO satellites can be compromised in such a way it doesn't constitute any power loads or difficulties. On the other hand it can improve the LEO coverage area so as not to be limited to its conventional ground station. This means that the available satellite - satellite time is enhanced to reach the percentage of $\mathbf{5 8 8 2 / 2 3 8 7}=\mathbf{4 5 \%}$, so there will be a direct communication between the two satellites for the length of almost half satellite period. The results from the matlab program shows that the availability of the LEO satellite for establishing communication session with it within the coverage area of the GEO satellite coverage was higher than the availability that can be obtained through a normal LEO - ground station communication session. Establishing and validating such LEO-GEO crosslink can extend the range of LEO satellite reconnaissance without the obstacles that can arise from the need to the existence of LEO ground station in hostile or nonallied land. Besides, the data from the LEO satellite can be obtained at any moment within the coverage zone of the GEO satellite. Regarding the early warning technique, this cross-link can provide the fighter aircrafts with the updates events in the combat zone in real time or on the way to destination [2].

All matlab programs used in this paper is available upon request at mamas20@hotmail.com

\section{REFERENCES}

[1] Niel L. Brandel, William A. Watson, And Aaron Weinberg, NASA's Advanced Tracking and Data Relay Satellite System for the Years 2000 and Beyond, Proceedings of IEEE, Vol. 78, No.7, July 2000.

[2] Giuliano Berretta, Agostino De Agostini, and Antony Dickinson, The European Data Relay System: Present Concept and Future Evolution, Proceedings of IEEE, Vol. 78, No.7, July 1990.

[3] Dwayne R. Morgan, Ronald G. Streich, arton Bull, Charles Grant, NASA/GSFC/WFF Code584, irst TDRSS then Commercial GEO \& Big LEO and Now through LEO (2003).

[4] James R.Wertz, Wiley J. Larson, Space mission Analysis and Design,1999.

[5] Dennis Roddy, Satellite Communications, Third Edition, 2000.

[6] Saboquost, Satellite communication Networks, Chapter 5, 1999.

[7] R.Binder, S.D Huffman, I.Guarantz, P.A. ena (1987) Crosslink architectures for a multiple satellite systems, Proceeding of IEEE,92,No.1, PP74-82 Jan (2000).

[8]Egyptian Satellite NILESAT Brochure,(2001). 
[9] Panagiotis Tsiotras, Haijun Shen, Georgia Institute of Technology, Satellite Attitude Control and Power Tracking With Energy/Momentum Wheel (2002).

[10] Matlab Reference Manual, Release 16, (2003).

[11] E.A. soliet , M. A. soliman, Karim A., optimal antenna for LEO-GEO cross-link

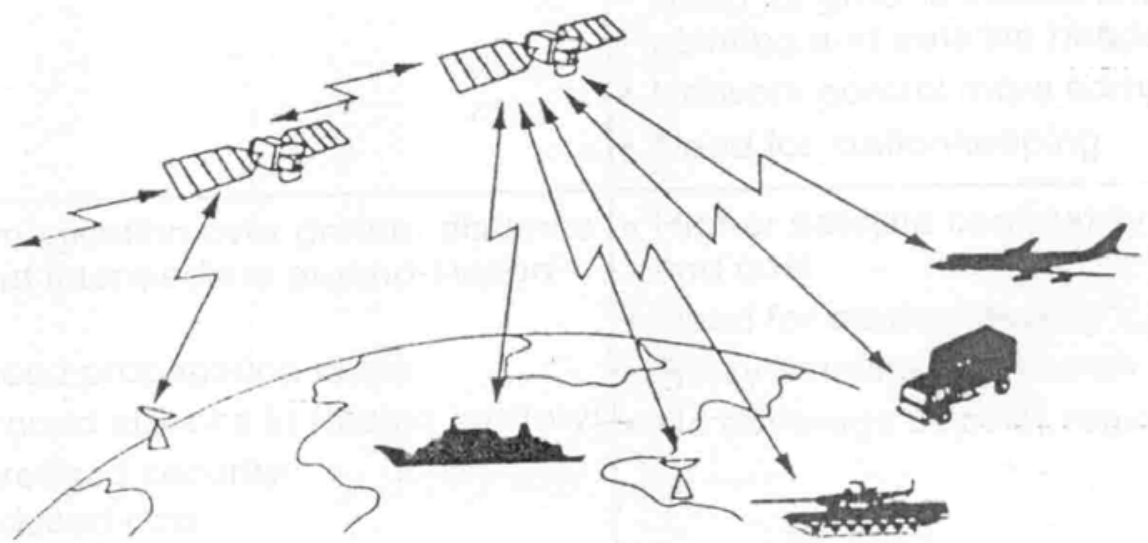

Fig 1 NILESAT as a Relay Satellite

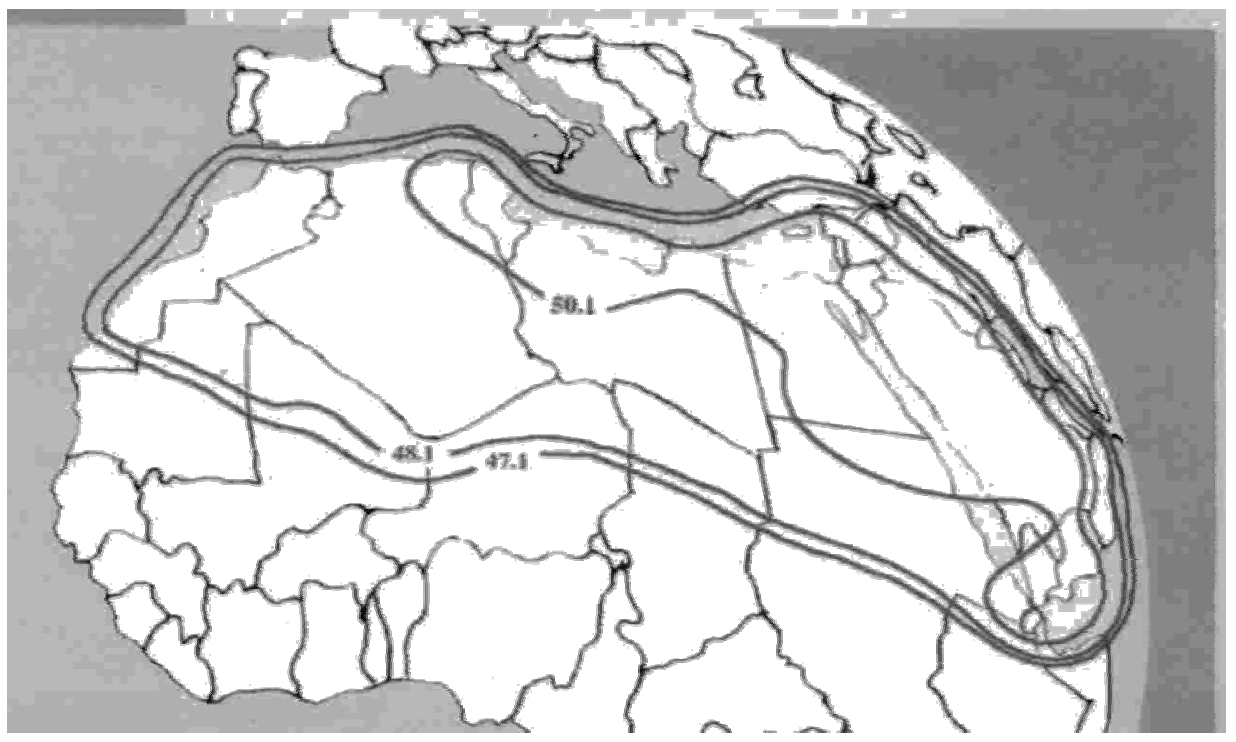

Fig 2 NILESAT Coverage Area Boundaries 


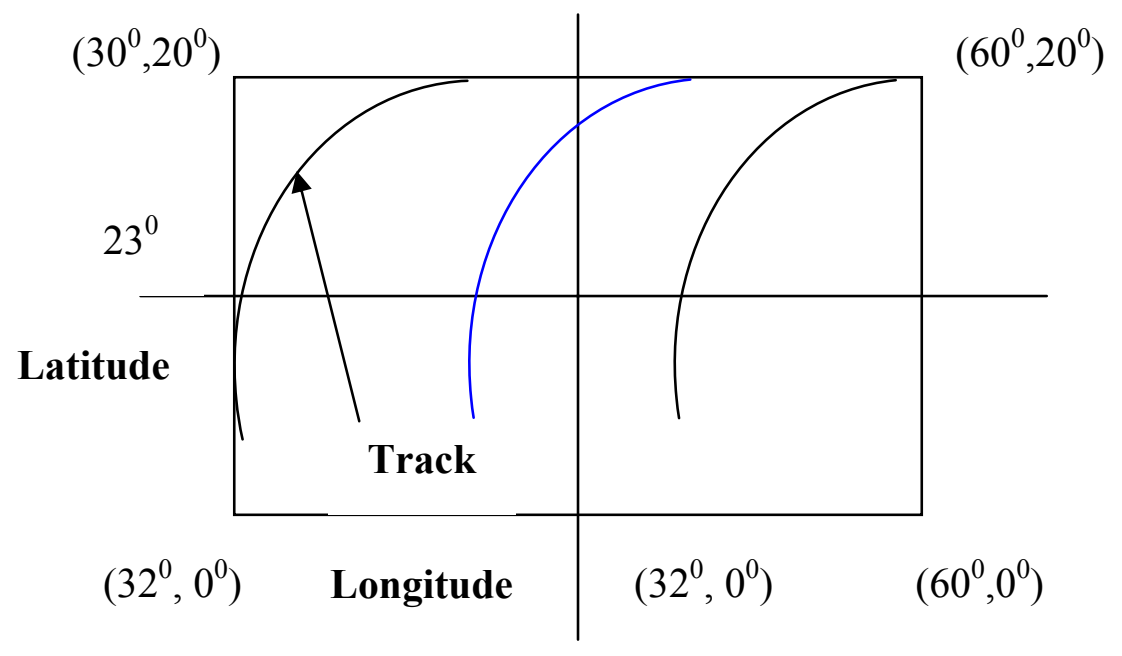

Fig 3 LEO satellite Ground Tracks Corners Covering the Local Regions (area of interest)

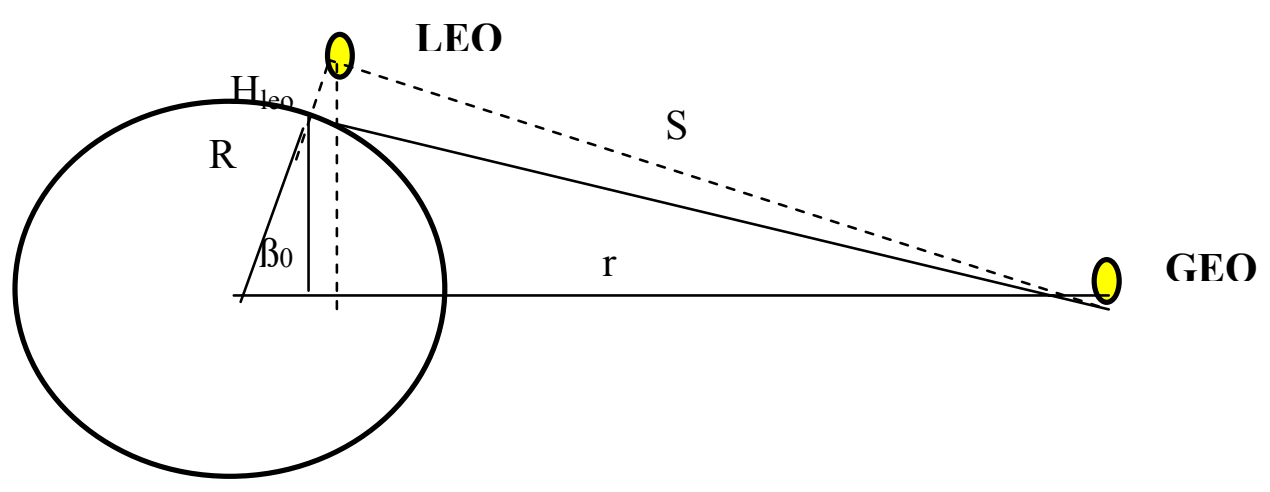

Fig 4 Propagation Path length calculation 


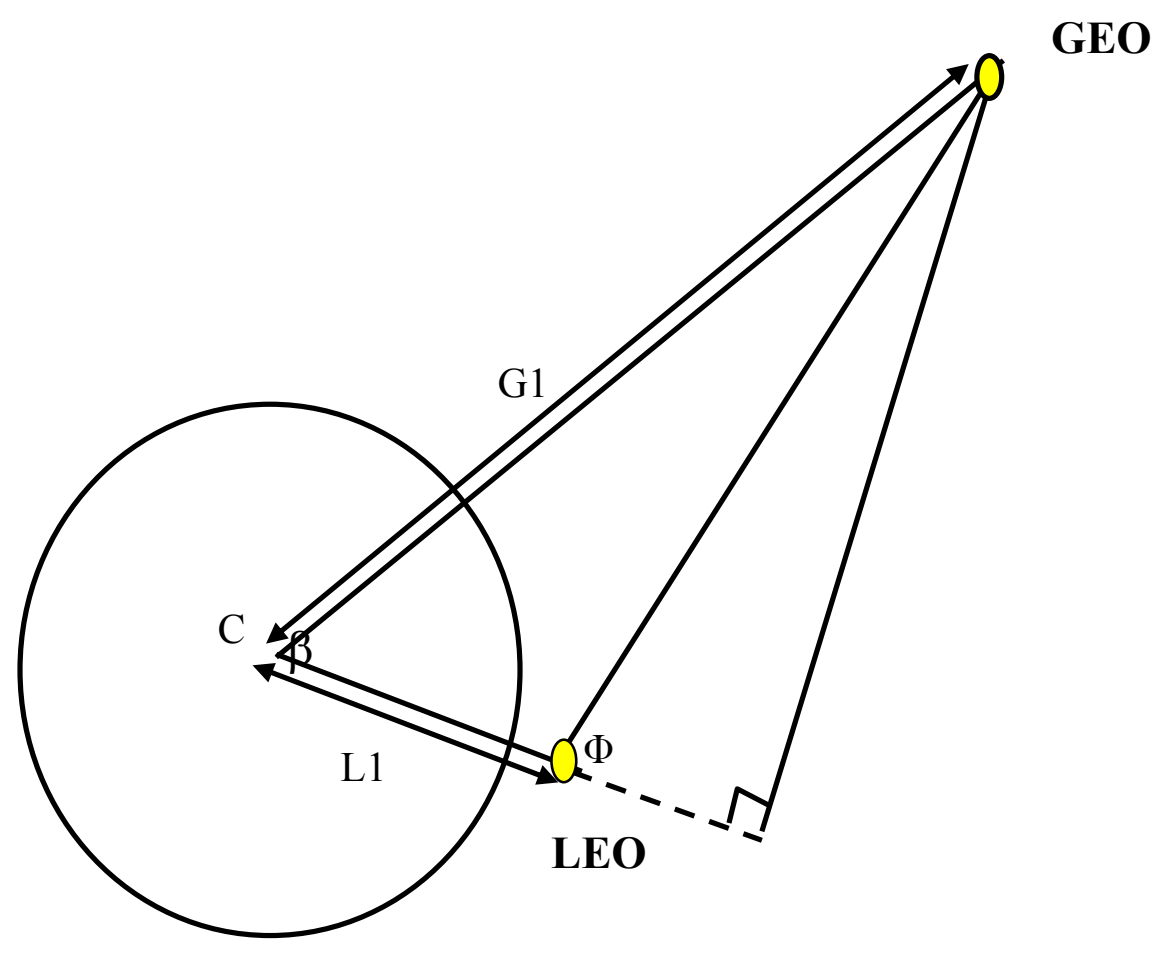

Fig 5 Cross Link Representation between GEO and LEO Satellites

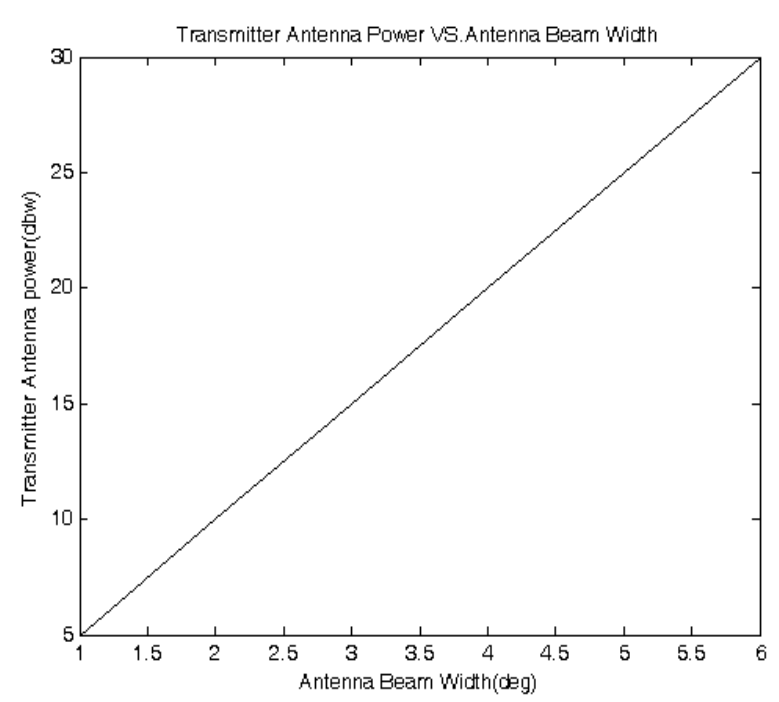

Fig 6 Transmitter Power Vs Antenna Beam width

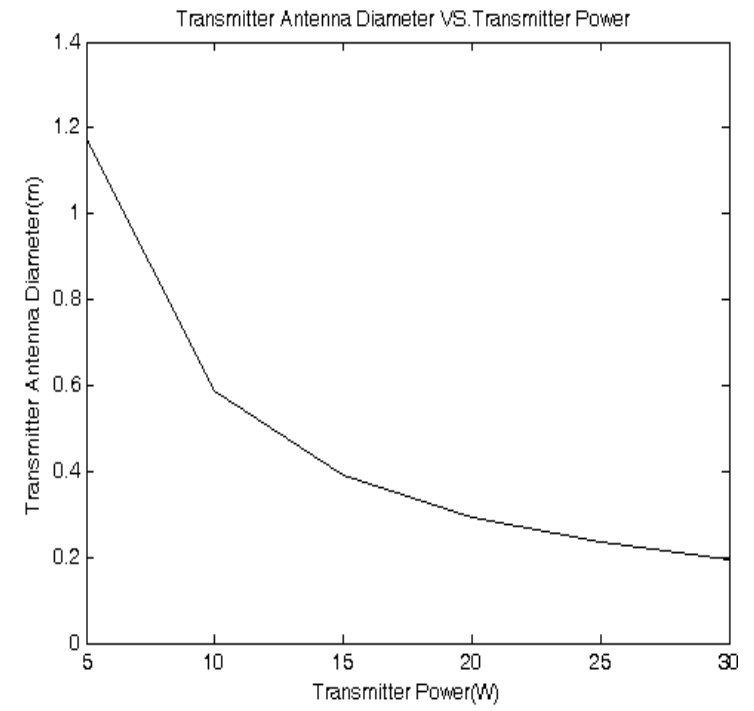

Fig 7 Transmitter Power Vs Antenna Diameter 


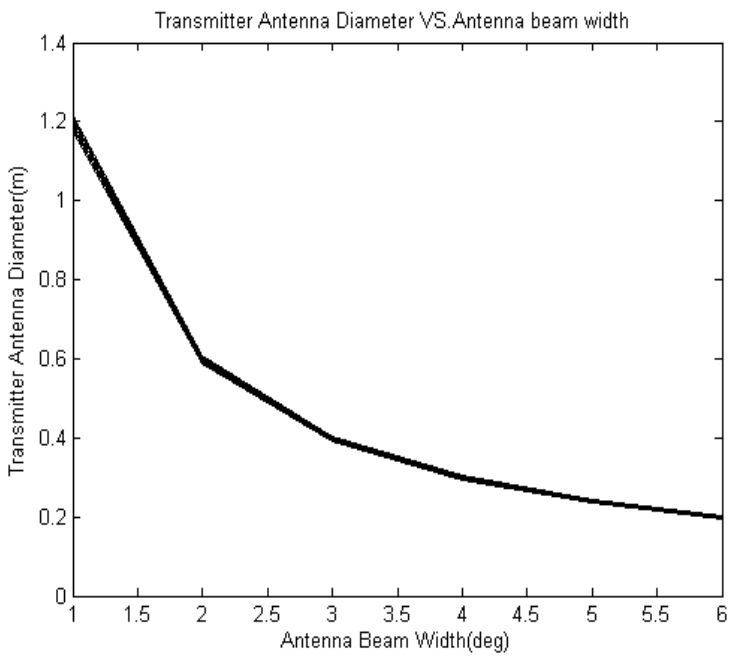

Fig 8 Transmitter Antenna Gain Vs Antenna Beam Width

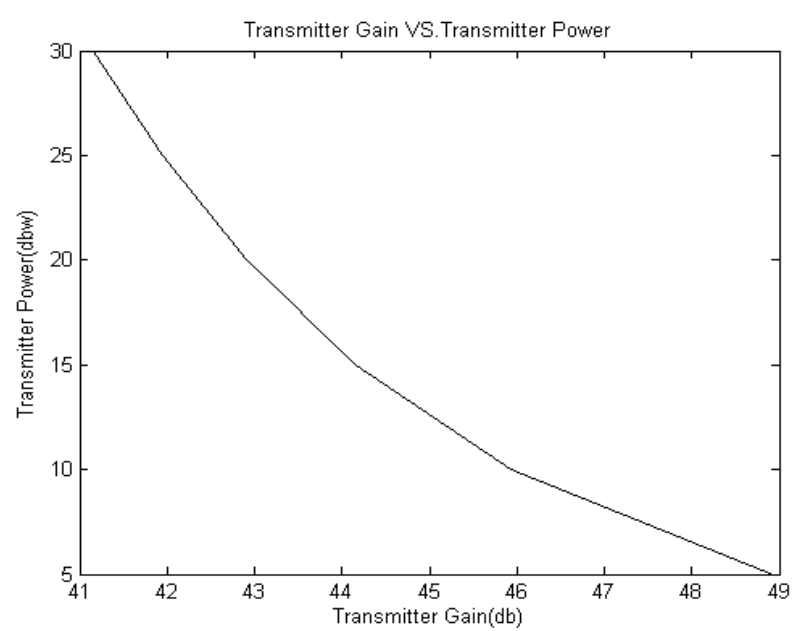

Fig 10 Transmitter Power Vs Transmitter Gain

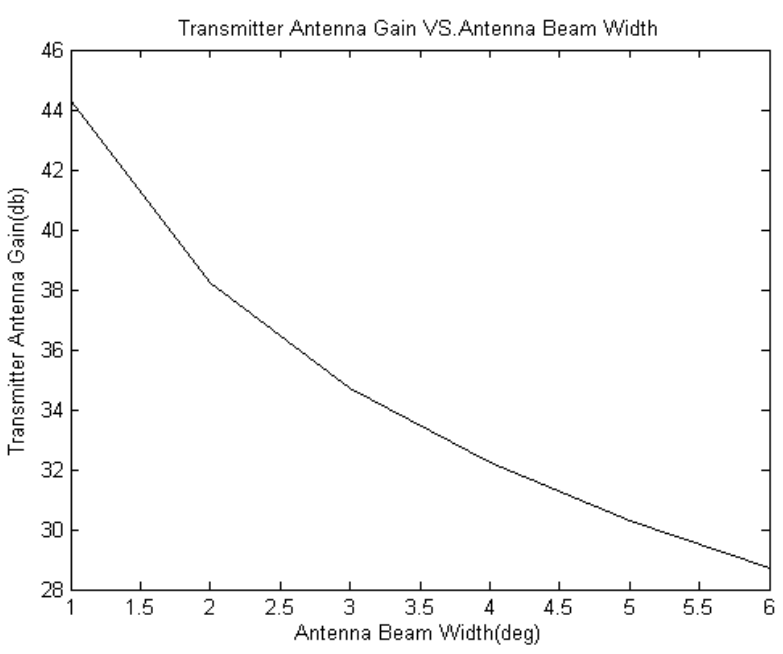

Fig 9 Transmitter Antenna Diameter Vs Antenna Beam Width

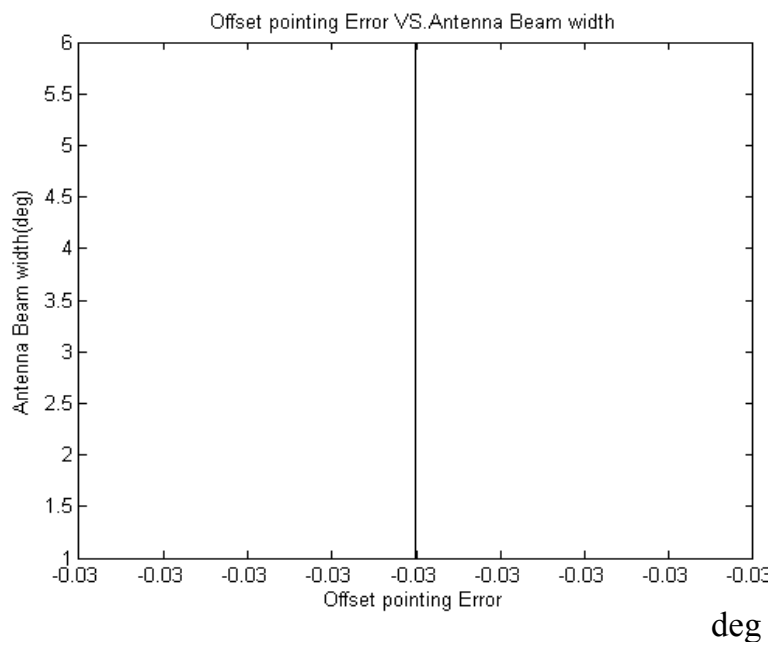

Fig 11 Offset Pointing Error Vs Antenna beam Width 


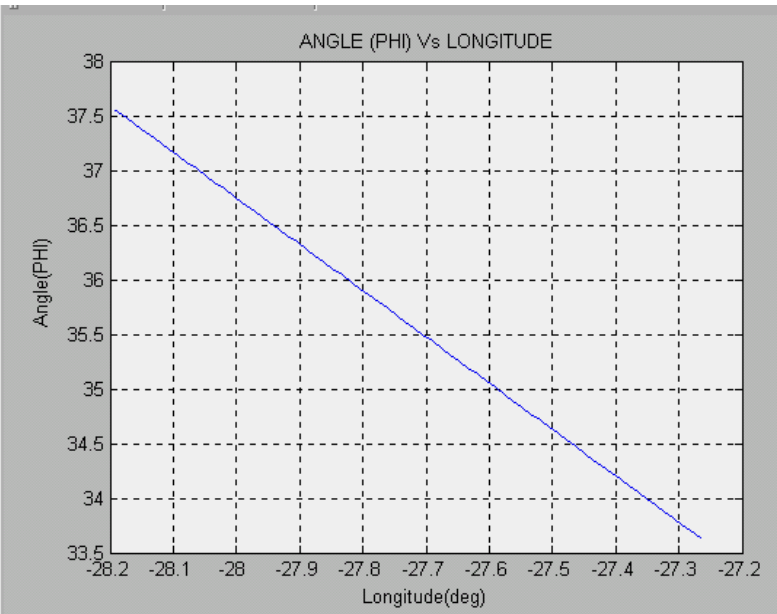

Fig 12 Angle (Ф) against Longitude

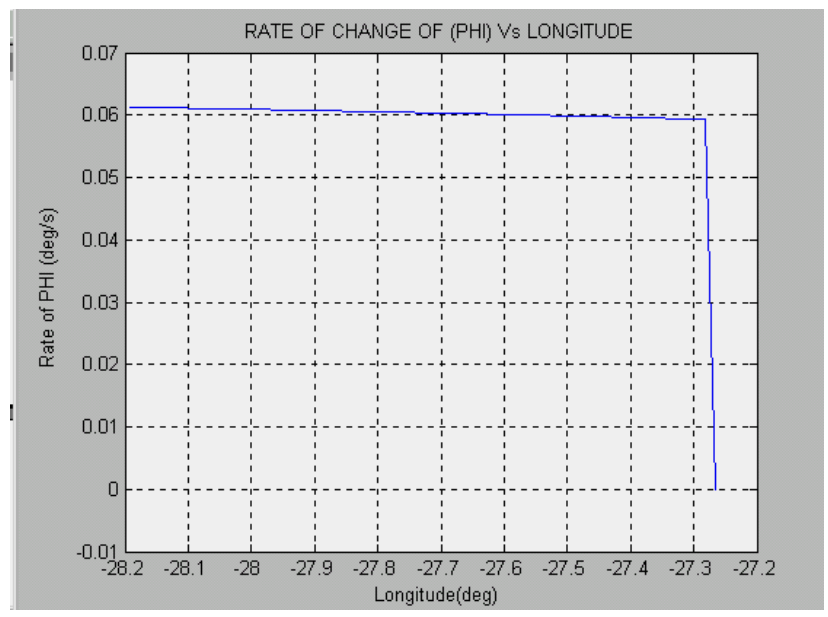

Fig 13 Rate of Change of $(\Phi)$ against Longitude

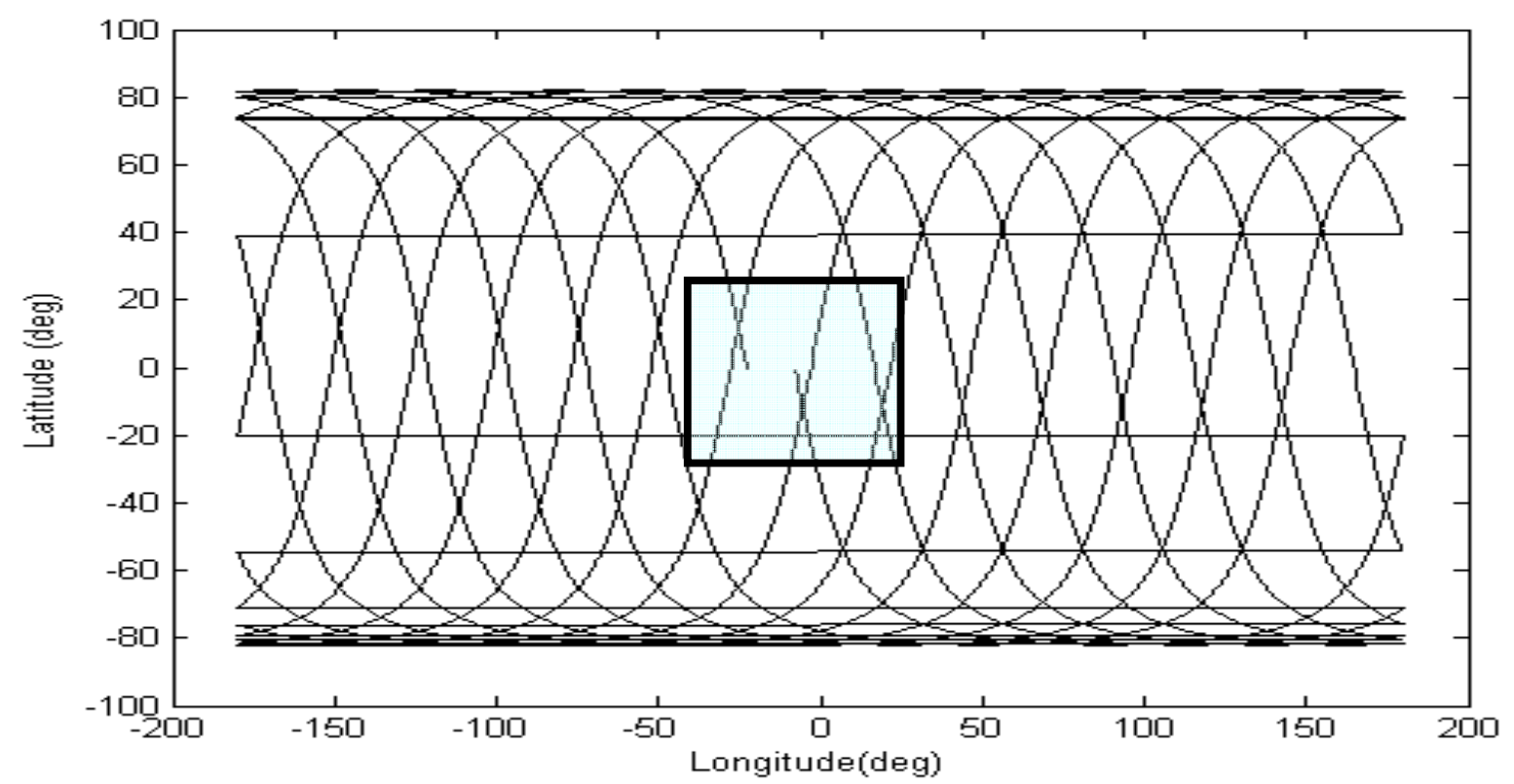

Fig 14 Ground Tracks of the Proposed LEO Satellite during a Day Period 